\title{
Dark-SUSY channels to study muon reconstruction performance at the Muon Collider
}

\section{Aimè ${ }^{a, b, *}$, C. Riccardi ${ }^{a, b}$, P. Salvini ${ }^{a, b}$ and I. Vai ${ }^{b, c}$ on behalf of the Muon Collider Physics and Detector working group}

${ }^{a}$ Physics Department, University of Pavia, Pavia, Italy

${ }^{b}$ Istituto Nazionale di Fisica Nucleare, Sezione di Pavia

Pavia, Italy

${ }^{c}$ Engineering and Applied Sciences Department, University of Bergamo

Dalmine (BG), Italy

E-mail: chiara.aime01@universitadipavia.it

In the context of simulation and reconstruction for the Muon Collider, muon reconstruction efficiency has been evaluated to explore the potential for the study of dark-SUSY channels.

In dark-SUSY models, supersymmetric particles act as a portal between Standard Model particles and the dark sector. In this analysis, the lightest Minimal Supersymmetric Standard Model neutralino decays, on one hand, directly in a dark photon or, on the other hand, in two dark photons through a dark Higgs boson. A muon pair, with kinematics driven by the photon mass, is then expected from each dark photon. Therefore, the final state is characterized by four muons in one channel and eight muons in the other.

Preliminary results of the muon reconstruction performance are shown for a possible range of neutralino and dark photon masses at a centre of mass energy of $3 \mathrm{TeV}$ for the time being without the effects of the machine Beam-Induced Background.

\footnotetext{
*** The European Physical Society Conference on High Energy Physics (EPS-HEP2021), ***

*** 26-30 July $2021 * * *$

*** Online conference, jointly organized by Universität Hamburg and the research center DESY ***
}

\footnotetext{
*Speaker
} 


\section{Introduction to dark-SUSY models}

Many theories have been proposed to solve Standard Model (SM) limitations such as the explanation of dark matter. Supersymmetry (SUSY), for example, is the generalization of the space-time symmetry of a quantum field theory that transforms fermions into bosons and viceversa [1]. In the so-called Minimal Supersymmetric Standard Model (MSSM) the number of superfields and interactions is kept as small as possible.

In dark-SUSY models, supersymmetric particles act as a portal between SM particles and the dark sector. In this analysis, a $\mathrm{U}(1)_{D}$ symmetry, broken at the $\mathrm{GeV}$ scale, has been added to the MSSM [2]. U(1) ${ }_{D}$ couples to the visible sector through kinetic mixing, implying the decay of the dark photon $a_{d}$ to SM leptons (e.g. two muons) and of the MSSM lightest neutralino $\tilde{N}_{1}$ to the hidden sector.

Two possible channels have been studied (Fig. 1): in the first $\tilde{N}_{1}$ decays directly in a dark photon, in the second $\tilde{N}_{1}$ decays through a dark Higgs boson $H_{d}$ in two dark photons. A muon pair (dimuon), with kinematics driven by the photon mass, is then expected from each dark photon giving final states characterized by four and eight muons, respectively. A dark neutralino $\left(n_{d}\right)$ is also produced resulting in missing energy.
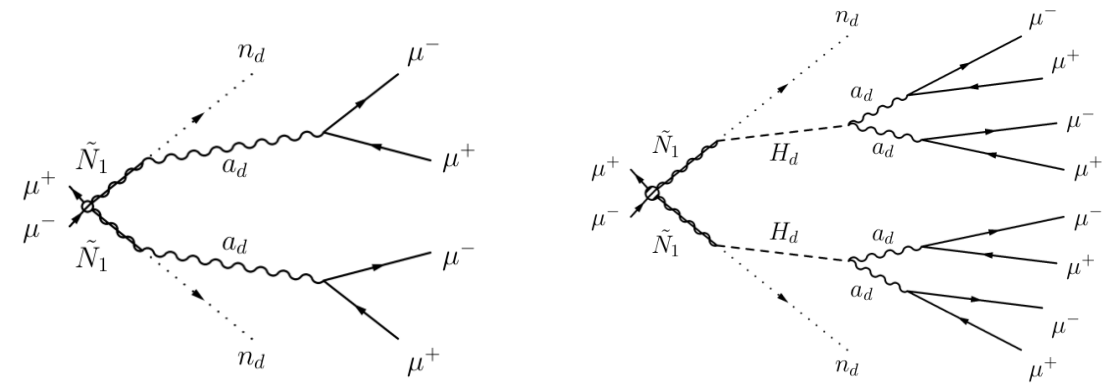

Figure 1: Dark-SUSY Feynman diagrams for channels with four (left) or eight muons (right) in the final state.

\section{Muon system at a Muon Collider}

A muon collider represents a promising proposal for high energy physics [3], probing much higher energy scales than hadrons colliding at the same energy, with a greatly reduced pile-up effect. The Beam-Induced Background (BIB), due to muon beams decay, is the only drawback.

A full simulation, currently based on CLIC ILC software [4], is ongoing to understand the performance of the detector. Focusing in particular on the muon system, CLIC design [5] foresees different layers (seven in the barrel and six in the endcaps) of glass Resistive Plate Chambers with $3 \times 3 \mathrm{~cm}^{2}$ cell size. Muon reconstruction is performed within the PANDORA PFA framework [6] and preliminary results [7] show a good single muon reconstruction efficiency for muons with transverse momentum greater than $10 \mathrm{GeV} / \mathrm{c}$, and BIB rejection.

In the following, reconstruction efficiency is evaluated for multi-muon final states at a centreof-mass energy of $3 \mathrm{TeV}$. 


\section{Preliminary results}

The analysis strategy is substantially based on the assumption that the two (four) dark photons in Fig. 1 decay in the same way and also the two dark Higgs bosons (Fig. 1 right).

Dimuons, as well as dark photon pairs in the eight muons channel, are collimated, but the kinematics is slightly different depending on the choice of masses. Dark photons in the four muons channel and dark Higgs bosons in the eight muons channel are back to back. Fig. 2 right shows, as an example, the angular distance $\left(\Delta R=\sqrt{\Delta \phi^{2}+\Delta \eta^{2}}\right.$, with $\phi$ the azimuthal angle and $\eta$ the pseudorapidity) for different particles in the eight muons channel.

For the channel with four muons, a selection is done requiring a difference in dimuon masses smaller than $5 \mathrm{GeV}$ and an angular distance greater than 1 . The invariant mass distribution of the dimuons passing these two cuts is fitted to obtain the reconstruction efficiency for the dark photons for different choices of neutralino and dark photon masses (Fig. 2 left).
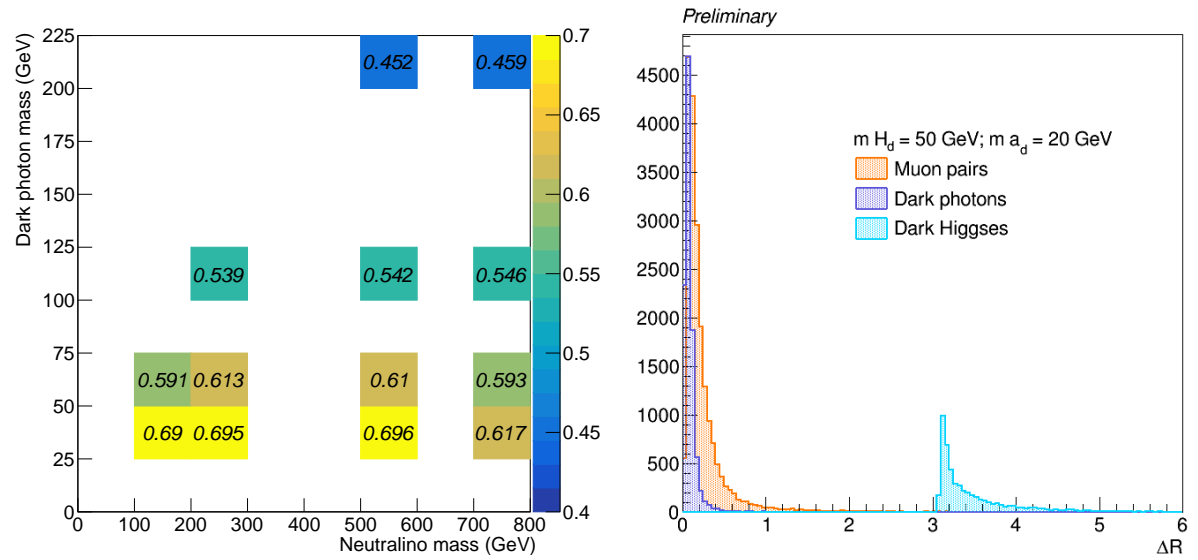

Figure 2: Left: reconstruction efficiency for four muons final state for different choices of neutralino and dark photon masses. Right: dimuons, dark photons and dark Higgs bosons pairs angular distance.

For the channel with eight muons, both similar dimuon masses and dark photon pair masses are required (percentage difference $<20 \%$ and $<10 \%$ respectively). The percentage of events passing a loose selection (number of reconstructed muons per event $\geq 8$ ) and a tight one (muons passing the two cuts above) are:

\begin{tabular}{||l||cc|cc|cc||}
\hline \multicolumn{1}{|c||}{} & \multicolumn{2}{c|}{$\mathrm{m}\left(\mathrm{H}_{\mathrm{d}}\right)=30 \mathrm{GeV} / \mathrm{c}^{2}$} & \multicolumn{2}{c||}{$\mathrm{m}\left(\mathrm{H}_{\mathrm{d}}\right)=50 \mathrm{GeV} / \mathrm{c}^{2}$} & \multicolumn{2}{c||}{$\mathrm{m}\left(\mathrm{H}_{\mathrm{d}}\right)=70 \mathrm{GeV} / \mathrm{c}^{2}$} \\
& loose & tight & loose & tight & loose & tight \\
\hline \hline $\mathrm{m}\left(\mathrm{a}_{\mathrm{d}}\right)=1 \mathrm{GeV} / \mathrm{c}^{2}$ & $34.5 \%$ & $32.2 \%$ & $33.0 \%$ & $30.5 \%$ & - & - \\
$\mathrm{m}\left(\mathrm{a}_{\mathrm{d}}\right)=10 \mathrm{GeV} / \mathrm{c}^{2}$ & $60.7 \%$ & $57.1 \%$ & $69.4 \%$ & $64.3 \%$ & $70.7 \%$ & $65.4 \%$ \\
$\mathrm{~m}\left(\mathrm{a}_{\mathrm{d}}\right)=20 \mathrm{GeV} / \mathrm{c}^{2}$ & - & - & $70.2 \%$ & $62.7 \%$ & $76.1 \%$ & $67.2 \%$ \\
$\mathrm{~m}\left(\mathrm{a}_{\mathrm{d}}\right)=30 \mathrm{GeV} / \mathrm{c}^{2}$ & - & - & - & - & $73.6 \%$ & $66.3 \%$ \\
\hline
\end{tabular}

The muon reconstruction efficiency, defined as the fraction of generated muons with a PANDORA track associated, is shown in Fig. 3 for different values of transverse momentum and polar angle for a dark Higgs boson of $50 \mathrm{GeV} / \mathrm{c}^{2}$ and different dark photon masses. Reconstruction efficiency for small dark photon mass $\left(1 \mathrm{GeV} / \mathrm{c}^{2}\right)$ is low because a high percentage of muons has a small transverse momentum $\left(p_{T}<10 \mathrm{GeV} / \mathrm{c}\right)$ or is outside the acceptance region $\left(12^{\circ}<\theta<168^{\circ}\right)$. 

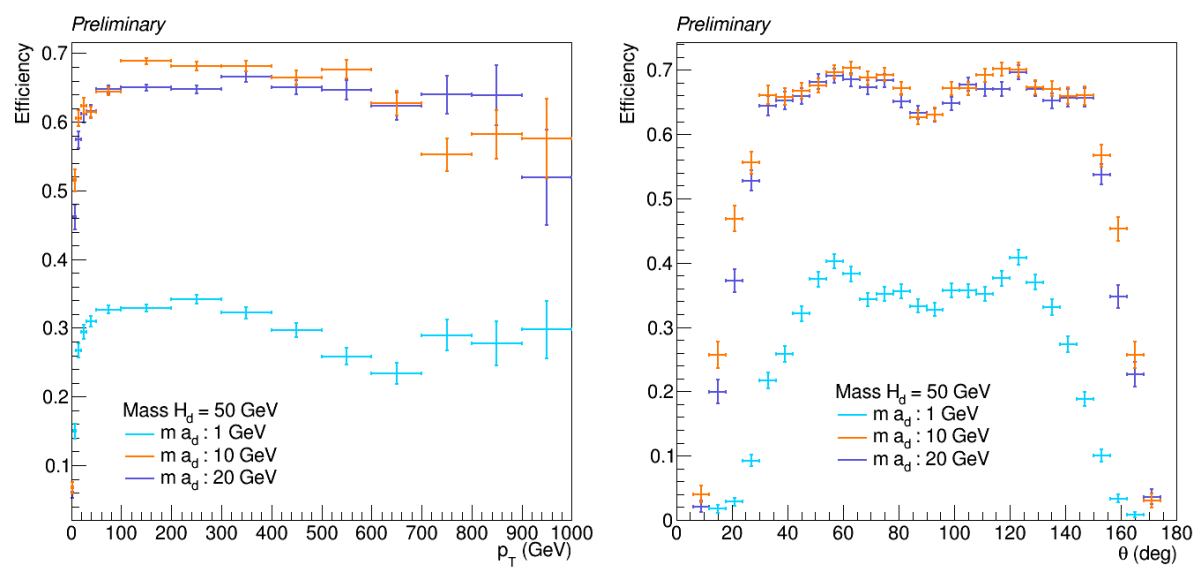

Figure 3: Muon reconstruction efficiency as a function of transverse momentum (left) and polar angle (right).

\section{Conclusions}

Muon reconstruction performance, based on PANDORA PFA framework, has been evaluated for dark-SUSY channels with multi-muon final states at a centre-of-mass energy of $3 \mathrm{TeV}$. Without the effects of the machine Beam-Induced Background, that slightly affects the muon system, a good muon reconstruction efficiency has been proved for a wide range of neutralino and dark photon masses.

\section{Acknowledgments}

This work was performed within the Muon Collider Detector Design and Performance group [8].

\section{References}

[1] M.Tanabashi et al., Review of Particle Physics, Phys. Rev. D 98, 030001 (2018).

[2] A.Falkowski et al., Hidden Higgs Decaying to Lepton Jets, J. High Energ. Phys. 2010, 77 (2010), [hep-ph/1002 .2952].

[3] K.R.Long et al., Muon colliders to expand frontiers of particle physics, Nat. Phys. 17, 289-292 (2021).

[4] ILCSoft, http://ilcsoft.desy.de/portal.

[5] L.Linssen et al.,Physics and Detectors at CLIC: CLIC Conceptual Design Report, CERN Yellow Report CERN-2012-003, [physics . ins-det/1202 . 5940].

[6] E. van der Kraaij and J. Marshall, Development of the PANDORA PFA NEW muon reconstruction algorithm, CERN LCD-Note-2011-004, 2011

[7] https://confluence.infn.it/display/muoncollider/Muon+detectors

[8] https://muoncollider.web.cern.ch/design/muon-collider-detector 